\title{
On the state estimation for dynamic power system
}

\author{
A. Thabet \\ Research Unit M.A.C.S \\ University of Gabes, TUNISIA
}

\author{
M. Boutayeb \\ Centre de Recherche CRAN \\ Nancy University, FRANCE
}

\author{
M.N. Abdelkrim \\ Research Unit M.A.C.S \\ University of Gabes, TUNISIA
}

\begin{abstract}
In this contribution we provide a simple and useful state estimation approach for a general class of non linear models that describe dynamic power systems. At first we show, through a small power network, that this class of systems is modeled by non linear differential-algebraic equations that we may always transform to a system of ordinary differential equations. After, we investigate a state estimator based on the EKF technique as well as the local stability analysis. High performances are illustrated through a simulation study applied on 3 and 5 buses test systems.
\end{abstract}

\section{Keywords}

Power system dynamics, State Estimation, Extended Kalman Filter, convergence analysis.

\section{Nomenclature}

M Inertia constant of the generator

D Damping constant of the generator

$\delta \quad$ mechanical rotor angle of the rotating machine

$\omega \quad$ mechanical angular velocity

$\omega_{s} \quad$ electrical angular velocity

$P_{M} \quad$ Mechanical power input

$P_{j}, Q_{j} \quad$ Nodal active and reactive power

$P_{c, d} \quad$ Transit power

$Y_{\text {bus }} \quad$ Nodal admittance matrix

$G_{i j}, B_{i j} \quad$ real and imaginary terms of bus admittance matrix corresponding to $i$ th row and $j$ th column

$N \quad$ Total number of system buses

$n_{g} \quad$ Number of generator buses

$n_{l} \quad$ Number of load buses

$P_{G i} \quad$ Electrical power supplied by the generator

\section{$\theta_{i}, V_{i} \quad$ Phase and voltage at bus $i$}

\section{INTRODUCTION}

Control design, diagnosis and monitoring of power systems become a major concern and an important economic issue during the last decades, in particular, in industrialized countries. Indeed, the main reasons are not only to optimize energy consumption but also to reduce the "Black Out" in vulnerable regions. It is important to observe, preferably in real-time, relevant variables of the power network. To achieve this goal it is necessary to use state estimation techniques or state observers from a database using physical sensors. Indeed, it is very difficult or impossible (for accessibility, technical and/or cost reasons) to measure through hardware sensors the excessive number of state variables in large scale systems. It is therefore important to develop software sensors that can produce a reliable estimate of the necessary variables. Unfortunately, these techniques are often based on a static model of the network around an operating point, and are off line due to large computational requirements. Therefore, the Energy Management Systems (EMS) based on these techniques are, in general, inefficient and lead to poor management of the networks [1].

State estimation in power system has mainly focused on Static State Estimation (SSE) from redundant measurement [2].

However, to oversee an electrical power system in efficient, economic and secure manner, it is most important to be acquainted with the different dynamics states, then, it is Dynamic State Estimation (DSE) in electric power system, which apprises of the aforesaid information.

In designing a DSE, it is important to model the dynamics of the complex bus voltages/phases (algebraic state) and generators (dynamic state). The existing models not only fail to depict the true behavior of the power system dynamics but also some of the parameters appearing in these models do not bear any physical meaning, reducing the size of model [3] or linearization form [4]. To override the limitations of the existing models, a relevant model has been considered in this paper to model the dynamics of the power system based on the nonlinear DAE models proposed in [5]. Where we show that we can always rewrite the system with a nonlinear DAE form with explicit ODE.

After the step of modeling, it is extremely important to consider a robust estimator which reflects a reliable image in the terms of capacity as for estimation, robustness and stability. The most important objective is the possibility of 
real-time application with the development of the Digital Signal Processor devices [6]. The existing methods are based on:

- The power system is considered as a quasi-static system and then applying a tracking estimator [7].

- Definition of spaces of linear combinations and their algebraic complement for the calculation of the observer gain [8].

- Consideration of linear approximation to extract the algebraic states [9] or to consider a linear dynamic power system model and applying the classical Kalman Filter [1] (by varying the algorithm of resolution such as Square Root Filter Algorithm [10] or changing the weight vector on measurement in objective function such as in the exponential form used in [11]).

We consider in this paper, an estimator based on a modified version of EKF while using new numerical approximations for the calculation of the Jacobian matrix.

The purpose of this work is to provide a generic dynamic model of balanced power systems and to design state estimation techniques to overcome the problems raised above. Indeed, description of the network by a dynamic model leads us to have an idea about the transient behavior that plays a central role for monitoring and control design. For doing so, through a 3 buses IEEE example, we show that the dynamic model is always written as a system of coupled dynamic nonlinear equations and algebraic ones. To develop useful and simple state estimators, we transform the obtained DAE model to an augmented one written as a system of ordinary differential equations. Thus, we propose a state estimator based on the Extended Kalman Filter, we also investigate stability analysis. In the last section, numerical simulations of two networks 3 and 5 buses test systems show the relevance and efficiency of the proposed approaches.

\section{DYNAMIC POWER SYSTEM MODEL}

The dynamics of a power system can be modeled with a combination of nonlinear differential equations and nonlinear algebraic equations. These sets of equations are often solved separately in different analysis techniques. The solution is accomplished in an iterative way, with the important feature that all the desired system characteristics are included. The general form of the DAE model is given as:

$$
\left\{\begin{array}{c}
\dot{\boldsymbol{x}}_{d}(t)=F_{d}\left(\boldsymbol{x}_{d}(t), \boldsymbol{x}_{a}(t), \boldsymbol{u}(t)\right) \\
0=g\left(\boldsymbol{x}_{d}(t), \boldsymbol{x}_{a}(t)\right) \\
\boldsymbol{y}(t)=h\left(\boldsymbol{x}_{d}(t), \boldsymbol{x}_{a}(t)\right)
\end{array}\right.
$$

With: $x_{d}(t) \in \square^{n_{d}}$ and $x_{a}(t) \in \square^{n_{a}}$ are respectively dynamic and algebraic states, $F_{d}(t) \in \square^{n_{d}}$ a function representing the nonlinear differential equations, $\quad g(.) \in \square^{n_{a}}$ represents the nonlinear algebraic constraints (equations), $\quad u(t) \in \square^{p}$ the control and $y(t) \in \square^{m}$ the output system. The problem with the system (1) is that $\dot{x}_{a}(t)$ does not appear explicitly.

\subsection{Problem Formulation}

To put out, in details, the physical dynamic power model, we will treat the case of the 3 buses test system given in Fig. 1 (with $n_{g}=2$ and $n_{l}=1$ ):

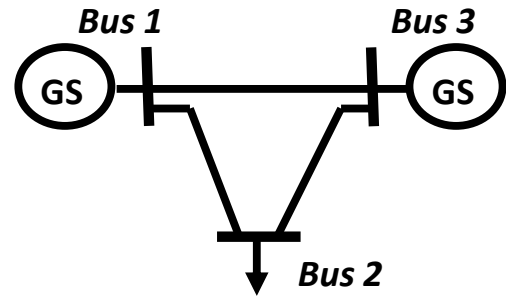

Figure 1.3 buses test system

We consider these assumptions [5]:

- The internal field currents are constant, providing the representation of the machine as a constant voltage behind the direct axis transient reactance.

- The mechanical power provided by the prime mover is constant and all dynamics of the prime mover are neglected.

- All generators are rotating at synchronous speed (steady state) and are round rotors.

- All generators in the system are identical, and therefore the inertia constant $\left(M_{i}\right)$ along with the damping constant $\left(D_{i}\right)$ of each generator have the same value.

- The mechanical rotor angle is the same as the electrical phase angle of the voltage therefore $\delta$ now refers to the electrical angle. To further simplify the notation, the transient reactance is incorporated into the system $Y_{\text {bus }}$, resulting in $\theta_{i}$ as generator terminal voltage phase and $V_{i}$ as the terminal voltage magnitude.

If we take node 1 as reference, the set of equation of this network is given by [8]:

$$
\left\{\begin{array}{c}
f_{i}^{I}: \dot{\delta}-\omega_{i}+\omega_{s}=0 \\
f_{i}^{I I}: \omega_{i}=\frac{\omega_{s}}{2 M}\left(P_{M_{i}}-P_{G_{i}}(\delta, \theta, V)-D \omega_{i}\right) \\
g_{i}^{I}: P_{j}-P_{j}(\delta, \theta, V)=0 \\
g_{i}^{I I}: Q_{j}-Q_{j}(\delta, \theta, V)=0 \\
y_{q}: P_{c, d}=P_{c, d}(\delta, \theta, V)
\end{array}\right.
$$

With:

$i=1 \ldots n_{g}-1 ; j=\left(n_{g}+1\right) \ldots\left(n_{g}+n_{l}\right) ; q=1 \ldots m ; c, d=1 \ldots N$, the node 1 is taken as the reference and :

$$
P_{G_{i}}=\sum_{j=1}^{N}\left|V_{i} \| V_{j}\right|\left[G_{i j} \cos \left(\delta_{i}-\theta_{j}\right)+B_{i j} \sin \left(\delta_{i}-\theta_{j}\right)\right]
$$

Therefore the model (2) can be rewritten under this form:

$$
\begin{gathered}
F(\dot{x}, x, \beta)=u \\
y=h(x, \beta)
\end{gathered}
$$


with:

$$
x=\left[\delta_{i}, \omega_{i}, \theta_{i}, V_{i}\right]^{T}, u=\frac{P_{M_{i}}}{M}, \beta=\left\{Y_{b u s}\right\}, F(.)=\left[f_{i}, g_{j}\right]^{T}
$$

and $y=P_{c, d}$ where $u$ and $y$ will be respectively the control and the output of the system. The choice of transit power as output which is based on this measure is used by the Tunisian Company of Electricity and Gas. Thus for this network, the state vector and the system equations are given by (3) and (4).

$$
x=\left[\begin{array}{llll}
x_{1} & x_{2} & x_{3} & x_{4}
\end{array}\right]^{T}=\left[\begin{array}{llll}
\delta_{3} & \omega_{3} & \theta_{2} & V_{2}
\end{array}\right]^{T}
$$

$$
\left\{\begin{array}{cc}
f^{I}: & \dot{x}_{1}=x_{2}-\omega_{s} \\
f^{I I}: & \dot{x}_{2}=\frac{\omega_{s}}{2 M}\left(P_{M_{3}}-P_{G_{3}}\left(x_{1}, x_{3}, x_{4}\right)-D x_{2}\right) \\
g^{I}: & P_{2}-P_{2}\left(x_{1}, x_{3}, x_{4}\right)=0 \\
g^{I I}: & Q_{2}-Q_{2}\left(x_{1}, x_{3}, x_{4}\right)=0 \\
y_{1}: & P_{3,2}\left(x_{1}, x_{3}, x_{4}\right)
\end{array}\right.
$$

with $x_{1}$ and $x_{2}$ are the dynamic variables, $x_{3}$ and $x_{4}$ are the algebraic variables. While using (1) the system is rewritten as:

$$
\left\{\begin{array}{c}
\dot{x}_{d}=\left(\begin{array}{c}
\dot{x}_{1} \\
\dot{x}_{2}
\end{array}\right)=F_{d}\left(x_{1}, x_{2}, x_{3}, x_{4}, u\right)=\left[f^{I}, f^{I I}\right]^{T} \\
g\left(x_{1}, x_{2}, x_{3}, x_{4}\right)=\left[g^{I}, g^{I I}\right]^{T}=0 \\
y(t)=P_{3,2}\left(x_{1}, x_{3}, x_{4}\right)
\end{array}\right.
$$

A simple diagram for the simulation of power system with model (1) is proposed, which: for the dynamic states we use a block of integration with nonlinear function $\left(F_{d}(t)\right)$ with algebraic constraints resolver under a package SIMULINK of $M A T L A ® B$. The simulation diagram is as follows (Fig. 2):

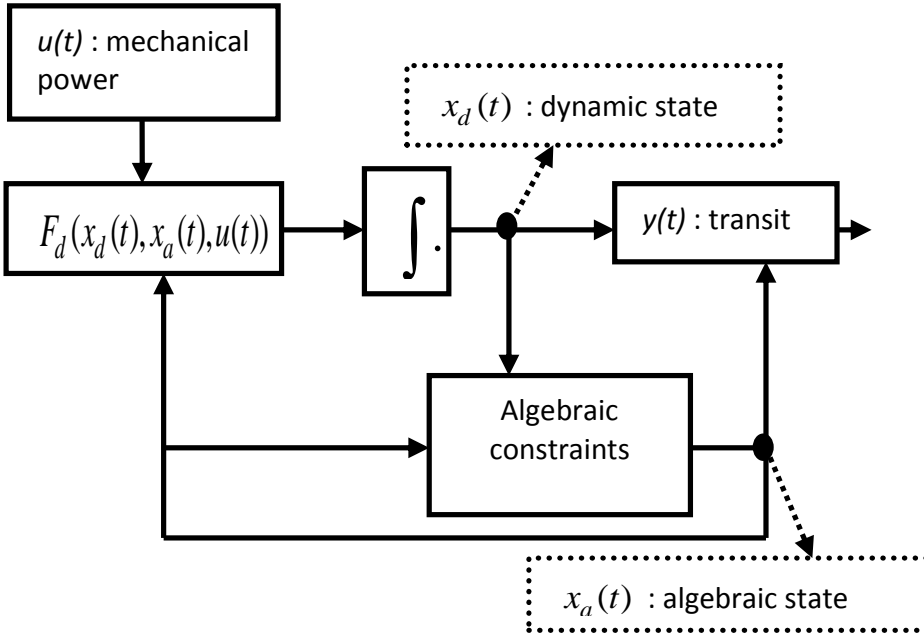

Figure 2. Diagram of simulation

\subsection{Semi-explicit DAE index 1}

If at an equilibrium point, the system (1) is called semiexplicit [9], index-1 property requires that $g\left(x_{d}, x_{a}\right)$ is solvable for $x_{a}$ and $\operatorname{det}\left(g_{x_{a}}\left(x_{d}, x_{a}\right)\right) \neq 0$ (to simplify $\left.x_{d}(t)=x_{d}, x_{a}(t)=x_{a}\right)$ :

$$
\left\{\begin{array}{c}
0=g_{x_{d}}\left(x_{d}, x_{a}\right) \dot{x}_{d}+g_{x_{a}}\left(x_{d}, x_{a}\right) \dot{x}_{a} \\
0=g_{x_{d}}\left(x_{d}, x_{a}\right) F_{d}\left(x_{d}, x_{a}, u\right)+g_{x_{a}}\left(x_{d}, x_{a}\right) \dot{x}_{a}
\end{array}\right.
$$

Where $g_{x_{a}}\left(x_{d}, x_{a}\right)=\frac{\partial g\left(x_{d}, x_{a}\right)}{\partial x_{a}}$ and

$g_{x_{d}}\left(x_{d}, x_{a}\right)=\frac{\partial g\left(x_{d}, x_{a}\right)}{\partial x_{d}}$ In other words, the differentiation index is 1 , if, by differentiation of the algebraic equations with respect to time, an implicit ODE system results [12]:

$\left\{\begin{array}{c}\dot{x}_{d}=F_{d}\left(x_{d}, x_{a}, u\right) \\ \dot{x}_{a}=-g_{x_{a}}{ }^{-1}\left(x_{d}, x_{a}\right) g_{x_{d}}\left(x_{d}, x_{a}\right) F_{d}\left(x_{d}, x_{a}, u\right)\end{array}\right.$

Where $g_{x_{a}}{ }^{-1}\left(x_{d}, x_{a}\right) \in \square^{n_{a} \times n_{a}}$ and $g_{x_{d}}\left(x_{d}, x_{a}\right) \in \square^{n_{a} \times n_{d}}$.

A study of nature and stability of DAE system is given by [13]. It should be noted that:

$g_{x_{a}}\left(x_{d}, x_{a}\right)=\frac{\partial g\left(x_{d}, x_{a}\right)}{\partial x_{a}}=\left(\begin{array}{ll}g_{x_{a} 1} & g_{x_{a} 2} \\ g_{x_{a} 3} & g_{x_{a} 4}\end{array}\right) \square[J]$

With $\boldsymbol{J}$ is the Jacobian matrix used in the Load Flow calculation excepted for generators terms, which allows us to verify that this $\operatorname{det}\left(g\left(x_{d}, x_{a}\right)\right) \neq 0$ and $\mathrm{g}$ is solvable for any $x_{a}$ (the elements of this matrix are the components of the diagonal Jacobian matrix used in load flow).

Finally, the complete model in form ODE is as follows:

$$
\begin{gathered}
\dot{x}=\left(\begin{array}{c}
\dot{x}_{d} \\
\dot{x}_{a}
\end{array}\right)=\bar{f}\left(x_{d}, x_{a}, u\right) \\
=\left(\begin{array}{c}
F_{d}\left(x_{d}, x_{a}, u\right) \\
-g_{x_{a}}^{-1}\left(x_{d}, x_{a}\right) g_{x_{d}}\left(x_{d}, x_{a}\right) F_{d}\left(x_{d}, x_{a}, u\right)
\end{array}\right) \\
\bar{y}=\left(\begin{array}{c}
0 \\
y
\end{array}\right)=\bar{h}\left(x_{d}, x_{a}\right)=\left(\begin{array}{l}
g\left(x_{d}, x_{a}\right) \\
h\left(x_{d}, x_{a}\right)
\end{array}\right)
\end{gathered}
$$

In the expression of $\bar{h}\left(x_{d}, x_{a}\right)$, the purpose of adding the algebraic constraint $g\left(x_{d}, x_{a}\right)$ is to check it permanently. It should be noted that the assumptions and the propositions given can be generalized for the other forms of dynamic power system models (models including a characteristic of the static/dynamic loads [14] and generators with exciter model [5]).

\section{DYNAMIC STATE ESTIMATION}

The main problem in dynamic state estimation of power system is that few methods are applicable. Effectively, the numerous and strong nonlinearities in presence lead generally to the use of Extended Kalman Filter to resolve the state estimation problem. We propose here the Extended Kalman Estimator to increase the precision as well as the 
robustness of the estimation. A study of the convergence of EKF will be presented.

\subsection{Extended Kalman Filter}

The Kalman filter is a recursive estimator. It means that to consider the running state, only preceding state and current measurements are necessary. The history of the observations and the estimates is; thus; not necessary. In the extended Kalman filter (EKF), the state transition and observation models need not be linear functions of the state but may instead be differentiable functions [15]. The considered nonlinear discrete system is given by (10):

$$
\left\{\begin{array}{c}
x_{k+1}=f\left(x_{k}, u_{k}\right)+v_{k} \\
y_{k}=h\left(x_{k}, u_{k}\right)+w_{k}
\end{array}\right.
$$

Where $v_{k}$ and $w_{k}$ are the system and observation noises which are both assumed to be zero mean multivariate Gaussian noises with covariance $Q_{k}$ and $R_{k}$ respectively.

In this paper, we have used the classical form of EKF (we have used Euler discretization with a step size $T e$, $x_{k+1}=x_{k}+T_{e} \bar{f}\left(x_{k}, u_{k}\right)=f\left(x_{k}, u_{k}\right)$ to discretize the continuous model (09)) given by:

$$
\begin{gathered}
\hat{x}_{k+1}=f\left(\hat{x}_{k}, u_{k}\right)+K_{k} e_{k} \\
K_{k}=F_{k} P_{k} H_{k}^{T}\left(H_{k} P_{k} H_{k}^{T}+R_{k}\right)^{-1} \\
P_{k+1}=\left(F_{k}-K_{k} H_{k}\right) P_{k} F_{k}^{T}+Q_{k} \\
e_{k}=y_{k}-h\left(\hat{x}_{k}, u_{k}\right)
\end{gathered}
$$

With:

$$
\begin{gathered}
F_{k}=F\left(\hat{x}_{k}, u_{k}\right)=\left.\frac{\partial\left(x_{k}+T_{e} \bar{f}\left(x_{k}, u_{k}\right)\right)}{\partial x_{k}}\right|_{x_{k}=\hat{x}_{k}} \text { and } \\
H_{k}=H\left(\hat{x}_{k}, u_{k}\right)=\frac{\partial \bar{h}\left(x_{k}, u_{k}\right)}{\partial x_{k}}=\left.\left(\begin{array}{c}
\frac{\partial g\left(x_{k}\right)}{\partial x_{k}} \\
\frac{\partial h\left(x_{k}\right)}{\partial x_{k}}
\end{array}\right)\right|_{x_{k}=\hat{x}_{k}} .
\end{gathered}
$$

There are some attempts to apply Kalman Filter on linearized D.A.E system [16], but our proposition is to apply the E.K.E in the classic general form with some numerical approximations that we propose for the Jacobian calculation.

Initially, it should be noted that due to the difficulty of finding $F_{k}$ (following the transformation of the algebraic variables in ODE model), we will make the following numerical approximation:

$$
\begin{gathered}
F_{k}=F\left(\hat{x}_{k}, u_{k}\right)=\left.\frac{\partial\left(x_{k}+T_{e} \bar{f}\left(x_{k}, u_{k}\right)\right)}{\partial x_{k}}\right|_{x_{k}=\hat{x}_{k}} \\
\frac{\partial\left(x_{d_{k}}+T_{e} F_{d}\left(x_{d_{k}}, x_{a_{k}}, u_{k}\right)\right)}{\partial\left(x_{d_{k}}, x_{a_{k}}\right)} \\
\left\{\begin{array}{c}
\frac{\partial\left(x_{a_{k}}+T_{e}\left(-g_{x_{a}}^{-1}\left(x_{d_{k}}, x_{a_{k}}\right) g_{x_{d}}\left(x_{d_{k}}, x_{a_{k}}\right) F_{d}\left(x_{d_{k}}, x_{a_{k}}, u_{k}\right)\right)\right)}{\partial\left(x_{d_{k}}, x_{a_{k}}\right)}
\end{array}\right\}
\end{gathered}
$$

The numerical approximation is used on the second term of $F_{k}$ (since it is very difficult to determine) as follows:

$$
\begin{gathered}
\frac{\partial\left(x_{a_{k}}+T_{e}\left(-g_{x_{a}}^{-1}\left(x_{d_{k}}, x_{a_{k}}\right) g_{x_{d}}\left(x_{d_{k}}, x_{a_{k}}\right) F_{d}\left(x_{d_{k}}, x_{a_{k}}, u_{k}\right)\right)\right)}{\partial\left(x_{d_{k}}, x_{a_{k}}\right)} \\
\approx\left(I_{n_{a}}+T_{e}\left(-g_{x_{a}}^{-1}\left(x_{d_{k}}, x_{a_{k}}\right) g_{x_{d}}\left(x_{d_{k}}, x_{a_{k}}\right) \frac{\partial F_{d}\left(x_{d_{k}}, x_{a_{k}}, u_{k}\right)}{\partial\left(x_{d_{k}}, x_{a_{k}}\right)}\right)\right)
\end{gathered}
$$

For $x_{d_{k}}=\hat{x}_{d_{k}}, x_{a_{k}}=\hat{x}_{a_{k}}$. The terms $g_{x_{a}}^{-1}$ and $g_{x_{d}}$ are calculated numerically.

\subsection{Convergence Analysis}

In this section, we present a convergence analysis of EKF based on the method of [17] [18] and [19] by including an unknown diagonal matrix to model linearization errors and a Lyapunov function. This is leads to the resolution of a LMI which depends on the choice on $R_{k}$ and $Q_{k}$.

Initially, the error vector is defined: $\tilde{x}_{k}=x_{k}-\hat{x}_{k}$ and the candidate Lyapunov function: $V_{k+1}=\tilde{x}_{k+1}^{T} P_{k+1}^{-1} \tilde{x}_{k+1}$, where :

$$
\left\{\begin{array}{c}
\tilde{x}_{k+1}=\alpha_{k}\left(F_{k}-K_{k} H_{k}\right) \tilde{x}_{k}=\alpha_{k} \tilde{F}_{k} \\
P_{k+1}^{-1}=\left(\tilde{F}_{k} P_{k} F_{k}^{T}+Q_{k}\right)^{-1} \\
\alpha_{k}=\operatorname{diag}\left(\alpha_{1 k}, \ldots, \alpha_{\left(n_{d}+n_{a}\right) k}\right)
\end{array}\right.
$$

We have then:

$$
\begin{aligned}
& V_{k+1}=\left(\alpha_{k} \tilde{F}_{k} \tilde{x}_{k}\right)^{T} P_{k+1}^{-1}\left(\alpha_{k} \tilde{F}_{k} \tilde{x}_{k}\right) \\
= & \tilde{x}_{k}^{T} \tilde{F}_{k}^{T} \alpha_{k}\left(\tilde{F}_{k} P_{k} F_{k}^{T}+Q_{k}\right)^{-1} \alpha_{k} \tilde{F}_{k} \tilde{x}_{k}
\end{aligned}
$$

A decreasing sequence $\left\{V_{k}\right\}_{k=1, \ldots}$ means that there exists a positive scalar $0<\xi<1$ so that: $V_{k+1}-(1-\xi) V_{k} \leq 0$. Therefore, this gives us this LMI:

$$
\tilde{F}_{k}^{T} \alpha_{k}\left(\tilde{F}_{k} P_{k} F_{k}^{T}+Q_{k}\right)^{-1} \alpha_{k} \tilde{F}_{k}-(1-\xi) P_{k}^{-1} \leq 0
$$

With the same reasoning used in [18], we determine domains in which (15) is satisfactory. Under the following assumption:

$\left|\alpha_{j k}\right| \leq \bar{\alpha}_{k}=\sup _{j}\left|\alpha_{j k}\right| \leq\left(\frac{(1-\xi) \underline{\sigma}\left(\tilde{F}_{k} P_{k} F_{k}^{T}+Q_{k}\right)}{\bar{\sigma}\left(F_{k}^{T}\right) \bar{\sigma}\left(P_{k}\right) \bar{\sigma}\left(\tilde{F}_{k}\right)}\right)^{\frac{1}{2}}$

$\left\{V_{k}\right\}_{k=1, \ldots}$ is a decreasing sequence. With $\underline{\sigma}$ and $\bar{\sigma}$ denoting the maximum and minimum singular values respectively, and as $\alpha_{k}$ is a diagonal matrix then:

$$
\begin{gathered}
{\left[\bar{\sigma}\left(\alpha_{k}\right)\right]^{2} \leq \frac{(1-\xi) \underline{\sigma}\left(\tilde{F}_{k} P_{k} F_{k}^{T}+Q_{k}\right)}{\bar{\sigma}\left(F_{k}^{T}\right) \bar{\sigma}\left(P_{k}\right) \bar{\sigma}\left(\tilde{F}_{k}\right)}} \\
\leq \frac{(1-\xi) \underline{\sigma}\left(P_{k}^{-1}\right)}{\bar{\sigma}\left(\tilde{F}_{k}^{T}\right) \bar{\sigma}\left(\left(\tilde{F}_{k} P_{k} F_{k}^{T}+Q_{k}\right)^{-1}\right) \bar{\sigma}\left(\tilde{F}_{k}\right)}
\end{gathered}
$$

We have then:

$$
\begin{gathered}
\bar{\sigma}\left(\tilde{F}_{k}^{T} \alpha_{k}\left(\tilde{F}_{k} P_{k} F_{k}^{T}+Q_{k}\right)^{-1} \alpha_{k} \tilde{F}_{k}\right) \\
\leq\left[\bar{\sigma}\left(\alpha_{k}\right)\right]^{2} \bar{\sigma}\left(\tilde{F}_{k}^{T}\right) \bar{\sigma}\left(\left(\tilde{F}_{k} P_{k} F_{k}^{T}+Q_{k}\right)^{-1}\right) \bar{\sigma}\left(\tilde{F}_{k}\right) \\
\leq(1-\xi) \underline{\sigma}\left(P_{k}^{-1}\right)
\end{gathered}
$$

When (18) is satisfied, $V_{k}$ is a strictly decreasing sequence. 
This last equation gives us an idea on the choice of $Q_{k}$ and for $R_{k}$, we proceed as follows:

$$
\begin{aligned}
& \bar{\sigma}\left(\tilde{F}_{k}\right)=\bar{\sigma}\left(F_{k}-K_{k} H_{k}\right) \\
& =\bar{\sigma}\left(F_{k}-F_{k} P_{k} H_{k}^{T}\left(H_{k} P_{k} H_{k}^{T}+R_{k}\right)^{-1} H_{k}\right) \\
& =\bar{\sigma}\left[F_{k}\left(I_{n_{a}+n_{d}}-P_{k} H_{k}^{T}\left(H_{k} P_{k} H_{k}^{T}+R_{k}\right)^{-1} H_{k}\right)\right]
\end{aligned}
$$

by replacing $P_{k} H_{k}^{T}\left(H_{k} P_{k} H_{k}^{T}+R_{k}\right)^{-1} H_{k}$ by $A_{k}$, we obtain:

$$
\begin{aligned}
& \bar{\sigma}\left(\tilde{F}_{k}\right) \leq \bar{\sigma}\left(F_{k}\right) \bar{\sigma}\left[A_{k}\left(A_{k}^{-1}+I_{n_{a}+n_{d}}\right)\right] \\
& \leq \bar{\sigma}\left(F_{k}\right) \bar{\sigma}\left(A_{k}\right) \bar{\sigma}\left(A_{k}^{-1}+I_{n_{a}+n_{d}}\right)
\end{aligned}
$$

with:

$$
\begin{aligned}
& \left.\bar{\sigma}\left(A_{k}\right) \leq \bar{\sigma}\left(P_{k} H_{k}^{T}\right) \bar{\sigma}\left(\left(H_{k} P_{k} H_{k}^{T}+R_{k}\right)^{-1} H_{k}\right)\right) \\
& \leq \bar{\sigma}\left(P_{k} H_{k}^{T}\right) \bar{\sigma}\left(\left(H_{k} P_{k} H_{k}^{T}+R_{k}\right)^{-1}\right) \bar{\sigma}\left(H_{k}\right) \\
& \leq \frac{\bar{\sigma}\left(P_{k} H_{k}^{T}\right) \bar{\sigma}\left(H_{k}\right)}{\underline{\sigma}\left(H_{k} P_{k} H_{k}^{T}+R_{k}\right)}
\end{aligned}
$$

We obtain finally:

$$
\bar{\sigma}\left(\tilde{F}_{k}\right) \leq \frac{\bar{\sigma}\left(F_{k}\right) \bar{\sigma}\left(P_{k} H_{k}^{T}\right) \bar{\sigma}\left(H_{k}\right) \bar{\sigma}\left(A_{k}^{-1}+I_{n_{a}+n_{d}}\right)}{\underline{\sigma}\left(H_{k} P_{k} H_{k}^{T}+R_{k}\right)}
$$

However, in order to ensure $\lim _{k \rightarrow \infty}\left(x_{k}-\hat{x}_{k}\right)=0$ and since $V_{k}$ is a strictly decreasing sequence and $P_{k}$ is a bounded matrix, it follows that:

$$
\begin{aligned}
& \qquad 0 \leq \mu \tilde{x}_{k}^{T} \tilde{x}_{k} \leq V_{k} \leq(1-\xi)^{k} V_{0} \\
& \Rightarrow 0 \leq \mu \lim _{k \rightarrow \infty}\left(\tilde{x}_{k}^{T} \tilde{x}_{k}\right) \leq \lim _{k \rightarrow \infty}\left(V_{k}\right) \leq V_{0} \lim _{k \rightarrow \infty}\left((1-\xi)^{k}\right)=0 \\
& \text { with } 0 \leq \mu I_{n_{d}+n_{a}} \leq P_{k}^{-1} .
\end{aligned}
$$

Consequently, in the same reasoning of [18] and [19], and to guarantee that the EKE ensures local asymptotic convergence, we must verify the following conditions:

- System (10) is A-locally uniformly rank observable, there exists $k \geq A-1$ where the observability matrix:

$$
\operatorname{rank}(O(k-A+1, k))=\left(n_{d}+n_{a}\right)
$$

where: $O(k-A+1, k)=\left[\begin{array}{c}H_{k-A+1} \\ H_{k-A+2} F_{k-A+1} \\ \cdots \\ H_{k} F_{k-1} \cdots F_{k-A+1}\end{array}\right]$

In practice, we use a numerical rank test on $O(k-A+1, k)$

- $F_{k}, H_{k}$ are uniformly bounded matrices and $F_{k}^{-1}$ exist.
- The matrices $Q_{k}$ and $R_{k}$ are chosen as follows:

$$
\begin{gathered}
Q_{k+1}=\gamma e_{k+1}^{T} e_{k+1} I_{n_{d}+n_{a}}+\lambda I_{n_{d}+n_{a}} \\
R_{k+1}=\varsigma H_{k+1} P_{k+1 / k} H_{k+1}^{T}+\tau I_{m}
\end{gathered}
$$

where $\gamma$ and $\lambda$ have to be chosen large and positive and $\varsigma$ and $\tau$ a positive scalar fixed by the user.

\section{SIMULATION RESULTS}

Studies are carried out on the IEEE 3 and 5 buses test system to evaluate the performance of the proposed dynamic model and the EKF. The transit power is considered as measurements. For the discretization of the model (09), we have used Euler discretization with a step size $T_{e}=10^{-3} \mathrm{~s}$.

\subsection{Results of simulation of 3 buses test system}

The measurement values are generated by adding low variance noise $( \pm 5 \%$ of real value) to the generated measurements (transit power $P_{3,2}$ ).

Firstly, to verify that $\operatorname{det}\left(g_{x_{a}}\left(x_{d}, x_{a}\right)\right) \neq 0$, Fig. 3 presents the evolution of $\operatorname{det}\left(g_{x_{a}}\left(x_{d}, x_{a}\right)\right)$ :

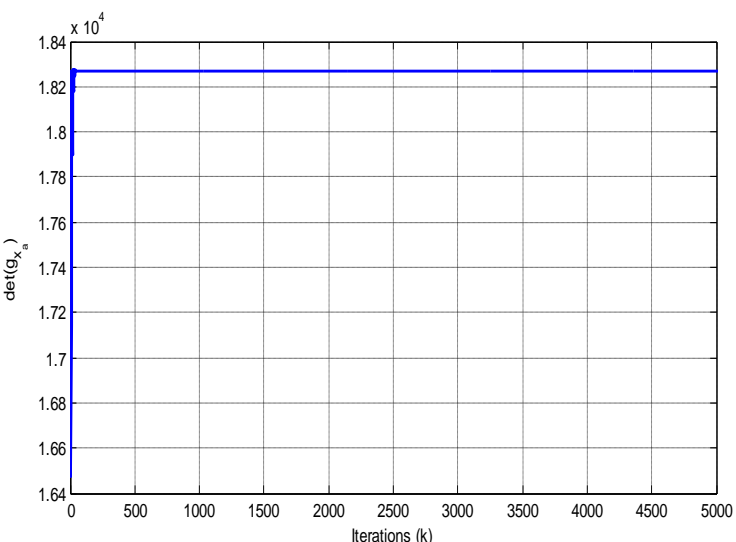

Figure 3. Evolution of $\operatorname{det}\left(g_{x_{a}}\left(x_{d}, x_{a}\right)\right)$.

Fig. 3 demonstrates in a clear way that the transformation of DAE model to ODE is applicable for power system.

Now, Fig. 4 shows the evolution of the rank of the observability matrix (numerical calculation with $A=4$ ).

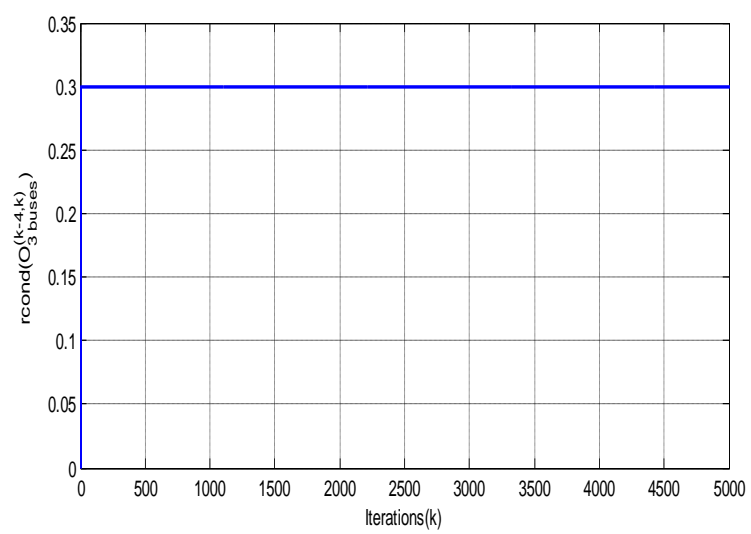

Figure 4. Evolution of $\operatorname{rcond}\left(O_{3 \text { buses }}^{(k-4, k)}\right)$. 
After the verification of the observability, $O_{3 \text { buses }}^{(k-4, k)}$ is well conditioned ( $\operatorname{rcond}\left(O_{3 \text { buses }}^{(k-4, k)}\right)>0$ ), we present the variation of $\left\|x^{\text {real }}-\hat{x}\right\|$ in Fig. 5 (based on the choice of $Q_{k}$ and $R_{k}$ ) to validate the convergence of the proposed estimator. We consider the measurement values are generated with the diagram of simulation (Fig. 2) and by adding high variance noise to the calculated measurements $( \pm 15 \%$ of real value of transit power $P_{3,2}$ ) with:

- Standard Choice of $Q_{k}$ and $R_{k}$ (Standard EKF):

$$
\begin{gathered}
Q_{k}^{E K F}=4.989 * 10^{-2} I_{4} \\
R_{k}^{E K F}=0.915
\end{gathered}
$$

- Proposed choice (Modified EKF):

$$
\begin{gathered}
Q_{k}^{E K F}=10^{10} e_{k}^{T} e_{k} I_{4}+10^{-3} I_{4} \\
R_{k}^{E K F}=10 H_{k} P_{k} H_{k}^{T}+10^{-3}
\end{gathered}
$$

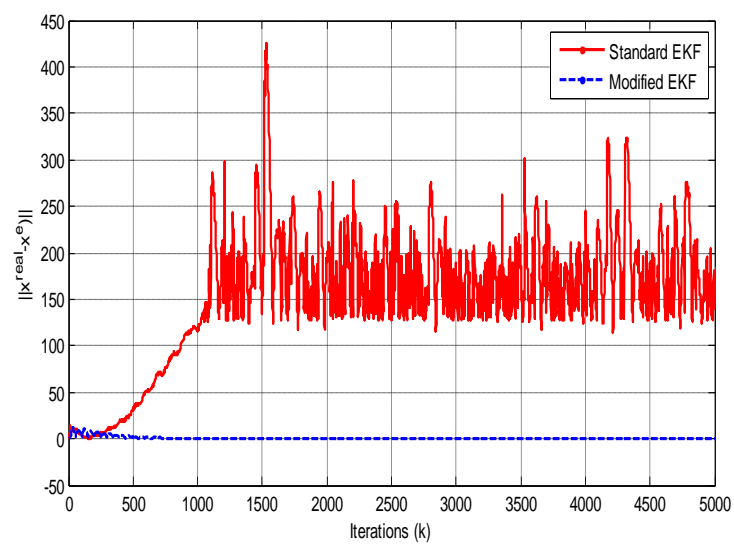

Figure 5. Evolution of $\left\|x^{\text {real }}-\hat{x}\right\|$.

Fig. 5 shows in a clear way, the interest of the appropriate choice of the expressions of $R_{k}$ and $Q_{k}$ to ensure stability and convergence. Another problem connected to the stability of DSE methods is the choice of initial values for various states to estimate. We tested the Standard and Modified EKF for 100 simulations while varying the initial values in a random way (variation of $\pm 20 \%$ with respect to the actual initial values). Table 1 shows the $\%$ of convergence by applying a disturbance to the system parameters:

- Case 1: Adding a low variance noise to the system (variation of $\pm 5 \%$ applied on $G_{i j}$ and $B_{i j}$ ).

- Case 2: Adding a high variance noise to the system (variation of $\pm 15 \%$ applied on $G_{i j}$ and $B_{i j}$ ).

Table 1. (\%) of Convergence with random initials values

\begin{tabular}{|r|l|l|}
\hline Estimator & \multicolumn{1}{|c|}{ Case 1 } & \multicolumn{1}{|c|}{ Case 2 } \\
\hline Standard EKF & $54 \%$ & $49 \%$ \\
\hline Modified EKF & $97 \%$ & $94 \%$ \\
\hline
\end{tabular}

In the general case, the studied algorithms converge to the good values only when are initialized \pm near to their actual values (the voltages are selected close to the values of the generators voltages and the phases equal to 0 ). Table 1 shows clearly that the Modified EKF converges in the majority of the cases compared with the Standard version.

\subsection{Results of simulation of 5 buses test system}

The network includes:

- 2 generators node: bus 1 and with node 1 is the reference bus and 3 static load nodes: 3,4 and 5 .

- The output is $\left(P_{2,3}\right)$ with a state vector composed by 8 variables: $[x]=\left[\delta_{2} \omega_{2} \theta_{3} V_{3} \theta_{4} V_{4} \theta_{5} V_{5}\right]^{T}$. All variables/sizes are given in p.u).

First, we present the evolution of the reciprocal condition estimator $\left(\operatorname{rcond}\left(O_{5 \text { buses }}^{(k-8, k)}\right)\right)$ :

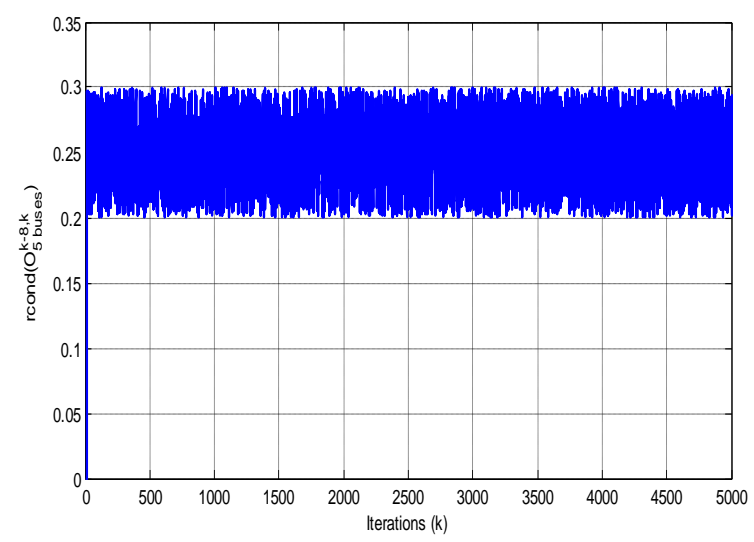

Figure 6. Evolution of $\operatorname{rcond}\left(O_{5 \text { buses }}^{(k-8, k)}\right)$

After the verification of the observability $\operatorname{rcond}\left(O_{5 \text { buses }}^{(k-8)}\right)$ is well conditioned $\left(\operatorname{rcond}\left(O_{5 b u s e s}^{(k-8, k)}\right)>0\right)$. We consider now, the measurement values are generated with the diagram of simulation (Fig. 2) and by adding high variance noise to the calculated measurements $( \pm 15 \%$ of real value of transit power $\left.P_{2,3}\right)$ with :

- Standard Choice of $Q_{k}$ and $R_{k}$ (Standard EKF):

$$
\begin{gathered}
Q_{k}^{E K F}=9.615 * 10^{-5} I_{8} \\
R_{k}^{E K F}=2.7 * 10^{-3}
\end{gathered}
$$

- $\quad$ Proposed choice (Modified EKF):

$$
\begin{gathered}
Q_{k}^{E K F}=10^{10} e_{k}^{T} e_{k} I_{24}+10^{-3} I_{8} \\
R_{k}^{E K F}=10 H_{k} P_{k} H_{k}^{T}+10^{-3}
\end{gathered}
$$

and we present the evolution of the norm of error estimation | $x^{\text {real }}-\hat{x} \|$ in Fig. 7: 


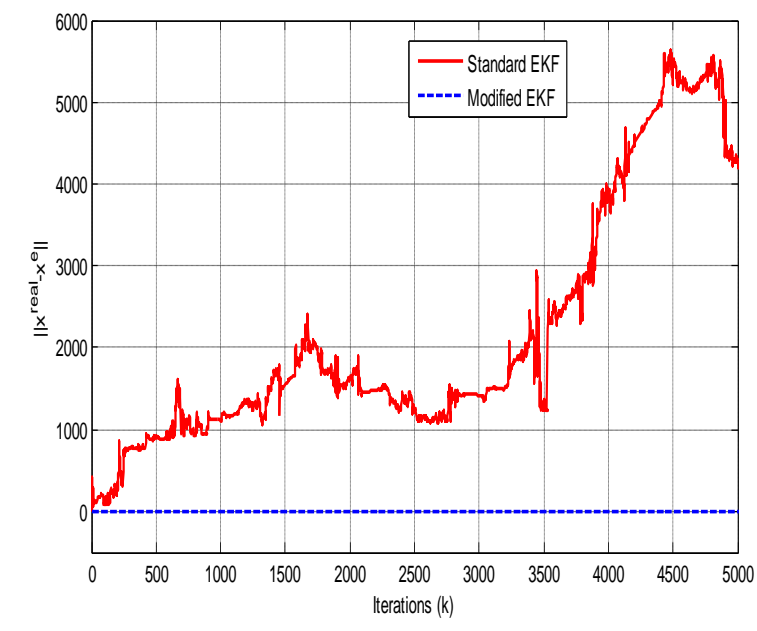

Figure 7. Evolution of $\mid x^{\text {real }}-\hat{x} \|$.

Fig. 7 shows that the appropriate choice of matrices $Q_{k}$ and $R_{k}$ with the proposed equation insures the convergence of the estimated states to the real values. This shows, very clearly, that the proposed estimator (including proposed numerical approximations for the calculation of Jacobian matrix) gives a reliable image in the terms of capacity as for estimation and robustness.

This is reflected by the existence of $\xi$ (given in Fig. 8) where the condition: $0<\xi<1$ is verified (section Convergence Analysis).

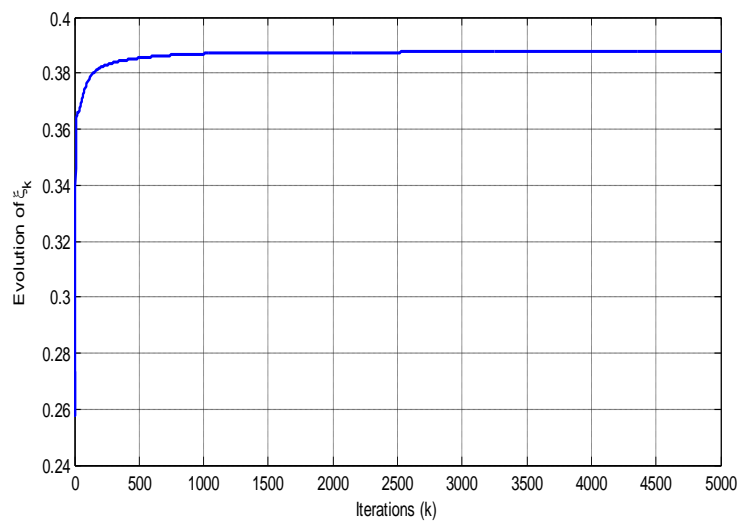

Figure 8. Evolution of $\xi_{k}$.

Fig. 8 shows the existence of $\xi_{k}$ which satisfies the condition already presented in the convergence analysis and is bounded between 0 and $1(\xi=0.39)$.

We present in Table 2 the $\%$ of convergence for 100 random initial values with Standard and Modified EKF (we consider the same two cases in 3 buses test system):

Table 2. (\%) of Convergence with random initial values

\begin{tabular}{|r|l|l|}
\hline Estimator & \multicolumn{1}{|c|}{ Case 1 } & \multicolumn{1}{|c|}{ Case 2 } \\
\hline Standard EKF & $53 \%$ & $46 \%$ \\
\hline Modified EKF & $96 \%$ & $93 \%$ \\
\hline
\end{tabular}

The values obtained in Table 2 confirm that the Modified EKF increases the estimation quality. In fact, the rate of convergence is multiplied by 2 times in comparison with Standard EKF.

It should be noted that the results of simulation of dynamic model given by the diagram (Fig. 2) are validated and compared with those generated by the Toolbox SimPowerSystems of MATLAB ${ }^{\circledR}$ (we obtained the same results). In addition, the use of this Toolbox facilitates the real time implementation in DSP device.

I one word, many results are omitted.

\section{CONCLUSION}

An efficient dynamic power system model has been described and investigated while based on introducing a transformation of ordinary DAE model. We also used the classical method of EKF to dynamic state estimation of power system while including some new numerical approximation for the calculation of Jacobian matrix and which was preceded by a convergence analysis. The results show well the appropriate choice of the dynamic model used in terms of robustness and, in a very clear way, the high quality of estimation offered by the Modified EKF. Experimental verification is then a necessity to testify the practical performance of this approach in the near future with real-time application for the monitoring and the diagnosis of large electrical network.

\section{REFERENCES}

[1] Shivakumar N. R. and Amit. 2008. A Review of Power System Dynamic State Estimation Techniques. In Power Syst. Technology and IEEE Power India Conf., India. $1-6$.

[2] Thabet A. et al. 2010. Power Systems Load Flow and State Estimation: Modified Methods and Evaluation of Stability and Speeds Computing. Int. Rev. of Electr. Eng., 5, 1110-1118.

[3] Scholtz E. 2004. Observer based monitors and distributed wave controllers for electromechanical disturbances in power systems. Doctoral Thesis, Massachusetts Institute Technology, USA.

[4] Pai M.A., Sauer P.W., Lesieutre B.C and Adapa R. 1995. Structural stability in power systems effect of load models. IEEE Trans. on Power Syst.10, 609-615.

[5] C.J. Dafis. 2005. An observbility formulation for nonlinear power systems modeled as differential algebraic systems. Ph.D. dissertation, Drexel university, PA, USA.

[6] Thabet A., Boutayeb M., Abdelkrim M.N.. 2012. Real time dynamic state estimation for power system. Int. J. of Computer Applications, 38, 11-18.

[7] Debes A.S. and Larson R.E. 1970. A dynamic estimator for tracking the state of a power system. IEEE Trans .on Power App. And Syst. 89, 1670-1678.

[8] Aslund J. and Frisk F. 2006. An observer for nonlinear differential-algebraic systems. Autmatica. 42, 959-965.

[9] Wichmann T. 2001. Simplification of nonlinear DAE systems with index tacking. In European Conf. on Circuit Theory and Design, Espoo, Finland, 173-176.

[10] Isabel M.F. and Barbosa F.P. 1994. Square root filter algorithm for dynamic state estimation of electric power systems. In Electrotechnical Conference, $7^{\text {th }}$ Mediterranean, Antalya, Turkey, 877-880. 
[11] Shih K. and Huang S. 2002. Application of a robust algorithm for dynamic state estimation of a power system. IEEE Trans. on Power Syst. 17, 141-147.

[12] B.W. Gordon. 2003. Dynamic sliding manifolds for realization of high index differential-algebraic systems. Asian J. of Control. 5, 454-466.

[13] D.C. Tarraf and H.H. Asada. 2002. On the nature and stability of differential-algebraic systems. Proc.American Control Conf., Anchorage, Al USA, 3546-3551.

[14] D. Karlsson and D.J. Hill. 1994. Modelling and identification of nonlinear dynamic loads in power systems. IEEE Trans. on Power Syst. 9, 157-166.

[15] K. Judd. 2003. Nonlinear state estimation, indistinguishable states, and the extended kalman filter. Physica D: Nonlinear Phenomena. 183, 273-281.
[16] V.M. Becerra, P.D.Roberts, and G.W. Griffiths. 2001 Applying the extended kalman filter to systems described by nonlinear differential-algebraic equations. Control Eng. Practice. 9, 267-281.

[17] M. Boutayeb and C. Aubry. 1999. A strong tracking extended kalman observer for nonlinear discrete-time systems. IEEE Trans. on Autom. Control. 44, 1550 1556.

[18] M. Boutayeb, (2000). Identification of nonlinear systems in the presence of unknown but bounded disturbances. IEEE Trans. on Autom. Control. 45, 1503-1507.

[19] Y. Song and J. Grizzle. 1995. The extended kalman filter as a local asymptotic observer for nonlinear discrete time systems. J. Math. Syst. Estimation and Control. 5, 5978. 